Draft VERSION OCTOBER 23, 2020

Typeset using LATEX RNAAS style in AASTeX63

\title{
Preliminary Target Selection for the DESI Emission Line Galaxy (ELG) Sample
}

\author{
Anand Raichoor, ${ }^{1}$ Daniel J. Eisenstein, ${ }^{2}$ Tanveer Karim, ${ }^{2}$ Jeffrey A. Newman, ${ }^{3}$ John Moustakas,${ }^{4}$ David D. Brooks, ${ }^{5}$ \\ Kyle S. Dawson, ${ }^{6}$ Arjun Dey, ${ }^{7}$ Yutong Duan, ${ }^{8}$ Sarah Eftekharzadeh, ${ }^{6}$ Enrique Gaztañaga, ${ }^{9,} 10$ Robert Kehoe, ${ }^{11}$ \\ Martin Landriau, ${ }^{12}$ Dustin Lang, ${ }^{13,14}$ Jae H. Lee, ${ }^{15}, 16$ Michael E. Levi, ${ }^{12}$ Aaron M. Meisner, ${ }^{7}$ Adam D. Myers, ${ }^{17}$ \\ Nathalie Palanque-Delabrouille, ${ }^{18}$ Claire Poppett, ${ }^{19}$ Francisco Prada, ${ }^{20}$ Ashley J. Ross, ${ }^{21}$ David J. Schlegel, ${ }^{12}$ \\ Michael Schubnell, ${ }^{22}$ Ryan Staten, ${ }^{11}$ Gregory Tarlé, ${ }^{22}$ Rita Tojeiro, ${ }^{23}$ Christophe Yèche, ${ }^{18}$ and Rongpu Zhou ${ }^{12,3}$ \\ ${ }^{1}$ Institute of Physics, Laboratory of Astrophysics, Ecole Polytechnique Fédérale de Lausanne (EPFL), Observatoire de Sauverny, 1290 \\ Versoix, Switzerland \\ ${ }^{2}$ Harvard-Smithsonian Center for Astrophysics, 60 Garden St., Cambridge, MA 02138 \\ ${ }^{3}$ University of Pittsburgh, 100 Allen Hall, 3941 O'Hara St., Pittsburgh, PA 15260, USA \\ ${ }^{4}$ Department of Physics 83 Astronomy, Siena College, 515 Loudon Road, Loudonville, NY 12211, USA \\ ${ }^{5}$ Department of Physics 85 Astronomy, University College London, Gower Street, London, WC1E 6BT, UK \\ ${ }^{6}$ Department of Physics and Astronomy, The University of Utah, 115 South 1400 East, Salt Lake City, UT 84112, USA \\ ${ }^{7}$ NSF's National Optical-Infrared Astronomy Research Laboratory, 950 N. Cherry Avenue, Tucson, AZ 85719, USA \\ ${ }^{8}$ Physics Department, Boston University, Boston, MA 02215, MA \\ ${ }^{9}$ Institute of Space Sciences (ICE, CSIC), 08193 Barcelona, Spain \\ 10 Institut d'Estudis Espacials de Catalunya (IEEC), 08034 Barcelona, Spain \\ ${ }^{11}$ Department of Physics, Southern Methodist University, 3215 Daniel Avenue, Dallas, TX 75275, USA \\ ${ }^{12}$ Lawrence Berkeley National Laboratory, 1 Cyclotron Road, Berkeley, CA 94720, USA \\ ${ }^{13}$ Perimeter Institute, Waterloo, ON N2L 2Y5, Canada \\ ${ }^{14}$ Department of Physics and Astronomy, University of Waterloo, 200 University Ave W, Waterloo, ON N2L 3G1, Canada \\ ${ }^{15}$ Department of Physics, Harvard University, Cambridge, MA 02138, USA \\ ${ }^{16}$ Vertex Pharmaceuticals, 50 Northern Ave., Boston, MA 02210, USA \\ ${ }^{17}$ Department of Physics 83 Astronomy, University of Wyoming, 1000 E. University, Dept 3905, Laramie, WY 8207 \\ ${ }^{18}$ IRFU, CEA, Université Paris-Saclay, F-91191 Gif-sur-Yvette, France \\ ${ }^{19}$ Space Sciences Laboratory at University of California, 7 Gauss Way, Berkeley, CA 94720 \\ ${ }^{20}$ Instituto de Astrofisica de Andalucía, Glorieta de la Astronomía, s/n, E-18008 Granada, Spain \\ ${ }^{21}$ Center for Cosmology and AstroParticle Physics, The Ohio State University, Columbus, OH 43212 \\ ${ }^{22}$ Department of Physics, University of Michigan, 450 Church St., Ann Arbor, MI 48109, USA \\ ${ }^{23}$ School of Physics and Astronomy, University of St Andrews, North Haugh, St Andrews, KY16 9SS, UK
}

Submitted to RNAAS

\begin{abstract}
DESI will precisely constrain cosmic expansion and the growth of structure by collecting 35 million redshifts across $\sim 80 \%$ of cosmic history and one third of the sky to study Baryon Acoustic Oscillations (BAO) and Redshift Space Distortions (RSD). We present a preliminary target selection for an Emission Line Galaxy (ELG) sample, which will comprise about half of all DESI tracers. The selection consists of a $g$-band magnitude cut and a $(g-r)$ vs. $(r-z)$ color box, which we validate using HSC/PDR2 photometric redshifts and DEEP2 spectroscopy. The ELG target density should be $\sim 2400 \mathrm{deg}^{-2}$, with $\sim 65 \%$ of ELG redshifts reliably within a redshift range of $0.6<z<1.6$. ELG targeting for DESI will be finalized during a 'Survey Validation' phase.
\end{abstract}

Keywords: Emission line galaxies, Surveys, Large-scale structures

\section{INTRODUCTION}

DESI (DESI Collaboration et al. 2016) will measure spectroscopic redshifts for $\sim 35$ million galaxies and quasars over $\sim 80 \%$ of cosmic history and one third of the sky. DESI will target multiple extragalactic tracers optimized for different redshift ranges, supplemented by significant stellar samples for calibration and Galactic science. Relaxed selections 


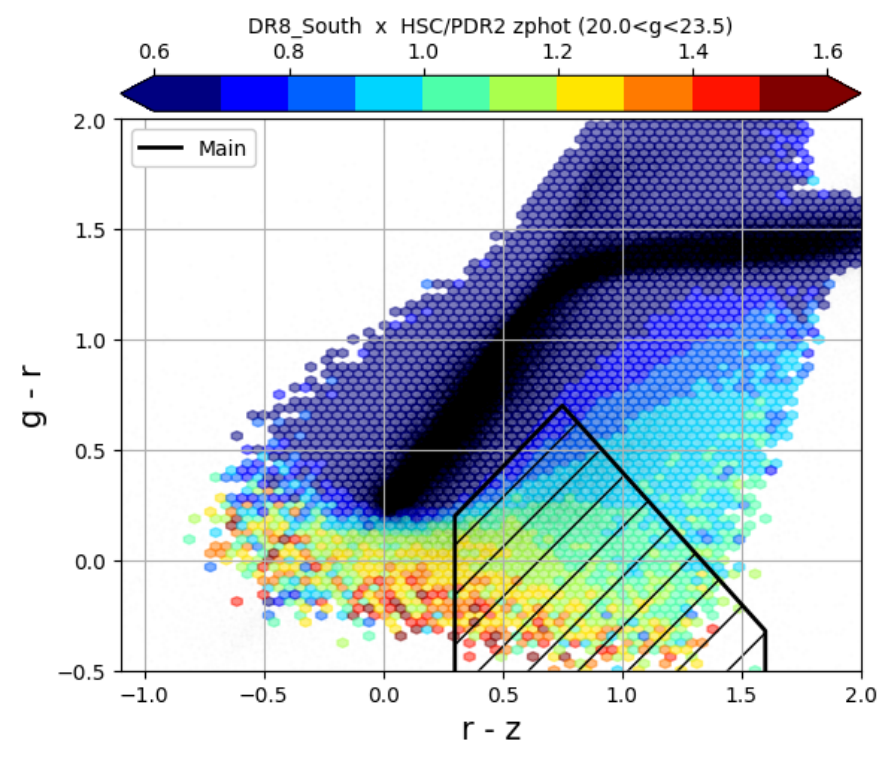

(a)

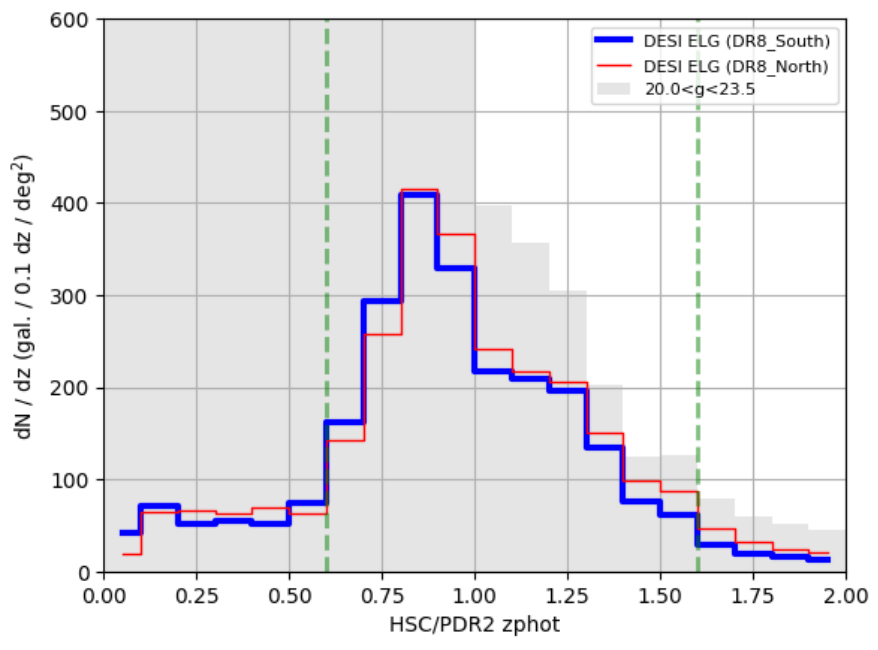

(b)

Figure 1. (a): $(g-r)$ vs. $(r-z)$ color-color diagram: the DESI ELG cuts for the 'South' are displayed using black hatched lines. The colored hexagons represent the mean redshift for $20.0<g<23.5$ sources using a photometric redshift $\left(z_{\text {phot }}\right)$ from the HSC/PDR2; photometric stars are displayed as black dots. (b): HSC/PDR2 $z_{\text {phot }}$ distribution for the DESI ELG targets, in the 'North' (red) and 'South' (blue). The gray shaded histogram shows the $z_{\text {phot }}$ distribution of the parent $20.0<g<23.5$ sample. Dashed green lines show the desired redshift range.

will be tested during a preliminary 'Survey Validation' phase to validate and optimize targeting for the DESI 'main' survey.

This note outlines preliminary targeting for an Emission Line Galaxy (ELG) sample in the redshift range $0.6<z<1.6$, which will constitute approximately half of DESI extragalactic tracers. DESI exploits the abundance of star forming galaxies at $z \sim 1-2$ to target ELG tracers with a spectroscopic redshift that can be measured reasonably quickly. The high star formation rate at these redshifts produces identifiable emission lines without the need to detect a strong continuum. A key spectroscopic diagnostic is the [O II] doublet at $\lambda \lambda$ 3726,3729: the DESI spectrographs are designed to resolve this feature over the targeted ELG redshift range. ELGs, which have been used as tracers in previous surveys (e.g., WiggleZ and eBOSS), will also underpin future BAO surveys, such as PFS (Takada et al. 2014) and Euclid (Laureijs et al. 2011).

\section{ELG TARGET SELECTION}

The target selection will use grz imaging from the Legacy Surveys (Dey et al. 2019). Results presented here are based on Data Release 8 of the Legacy Surveys ${ }^{1}$. The DESI footprint is split into two regions; 'North' (Galactic $b>0^{\circ}$ and Dec. $\left.>32.375^{\circ}\right)$ and 'South'; the 'North' has a slightly different photometric system and is shallower (0.5 magnitudes) in the $g$ - and $r$-bands. We therefore define slightly different cuts for the 'North' and 'South'.

First, we require a minimum photometric quality by enforcing at least one observation and a positive signal-to-noise ratio in each of $g_{-}, r_{-}$, and $z$-band. We also require that targets are not in corrupted pixels, nor near bright or medium-bright stars, globular clusters, or large galaxies (MASKBITS is not set for bits 1, 5, 6, 7, 11, 12 or 13).

${ }^{1}$ http://legacysurvey.org/dr8/ 
Next, we apply the following cuts in grz (see Figure 1):

$$
\begin{array}{r}
20.0<g<g_{\max } \\
0.3<(r-z)<1.6 \\
(g-r)<1.15 \times(r-z)+\mathrm{zpt} \\
(g-r)<-1.20 \times(r-z)+1.6,
\end{array}
$$

with $\left(g_{\max }, \mathrm{zpt}\right)=(23.6,-0.35)$ for the 'North' and $\left(g_{\max }, \mathrm{zpt}\right)=(23.5,-0.15)$ for the 'South'. All magnitudes are corrected for Galactic extinction using the Schlegel et al. (1998) maps. Eqs. (1b) and (1c) select targets in the desired redshift range and Eq. (1d) favors star-forming galaxies. As the photometry is noisier in the 'North', our selection box is farther from the low-redshift locus to avoid significant contamination from $z<0.6$ galaxies. Eq. (1a) targets the requisite [O II] flux (see e.g., Comparat et al. 2015) and also sets the density to $\sim 2400 \mathrm{deg}^{-2}$.

As no spectroscopic truth table exists for a complete sample with $g \lesssim 23.5$, we assess our selection using HSC/PDR2 DEmP photometric redshifts $\left(z_{\text {phot }}\right.$; Aihara et al. 2019) for the redshift distribution, and DEEP2 spectroscopic data over $0.8<z<1.4$ for the [O II] flux (Newman et al. 2013). The HSC/PDR2 $z_{\text {phot }}$ cover $\sim 100 \mathrm{deg}^{2}$ in the 'North' and $\sim 200 \mathrm{deg}^{2}$ in the 'South'. The $z_{\text {phot }}$ are estimated from deep grizy-photometry and are of exquisite quality for $z<1.6$ ELGs when compared to spectroscopy from eBOSS and from DESI Pilot Observations with the MMT (Raichoor et al. 2020; Karim et al. 2020). Figure 1 shows the $z_{\text {phot }}$ distribution of our ELG targets, demonstrating that $z_{\text {phot }}>1.0$ objects are efficiently selected; overall, $\sim 80 \%$ of the selection has $0.6<z_{\text {phot }}<1.6$ for both 'North' and 'South'.

Finally, we characterize [O II] flux for our selection using measurements from Comparat et al. (2015) in DEEP2 over $0.8<z<1.4$, where DEEP2 is complete over all fields for our $g<23.5-23.6$ ELG sample. We find that $76 \%$ ('North') and $83 \%$ ('South') of our targets have sufficient [O II] flux for a secure spectroscopic redshift measurement given the expected DESI specifications.

\section{CONCLUSION}

This note outlines a preliminary DESI ELG selection based on a $g$-band cut and a $(g-r)$ vs. $(r-z)$ color-color box that produces $\sim 2400 \mathrm{deg}^{-2}$ targets. Analyses using HSC/PDR2 $z_{\text {phot }}$ and DEEP2 [O II] flux show that $\sim 65 \%$ of resulting ELGs will provide a reliable spectroscopic redshift within $0.6<z<1.6$, in accord with DESI Collaboration et al. (2016). Preliminary ELG targeting will be tested during DESI 'Survey Validation' to inform a final selection for the DESI 'main' survey. Target catalogs that use the selection described in this note are public ${ }^{2}$.

AR acknowledges support from the ERC advanced grant LIDA and from the SNF grant 200020_175751. This research is supported by the Director, Office of Science, Office of High Energy Physics of the U.S. Department of Energy under Contract No.DE-AC02-05CH1123, and by the National Energy Research Scientific Computing Center, a DOE Office of Science User Facility under the same contract; additional support for DESI is provided by the U.S. National Science Foundation, Division of Astronomical Sciences under Contract No. AST-0950945 to the NSF's National OpticalInfrared Astronomy Research Laboratory; the Science and Technologies Facilities Council of the United Kingdom; the Gordon and Betty Moore Foundation; the Heising-Simons Foundation; the French Alternative Energies and Atomic Energy Commission (CEA); the National Council of Science and Technology of Mexico; the Ministry of Economy of Spain, and by the DESI Member Institutions. The authors are honored to be permitted to conduct astronomical research on Iolkam Du'ag (Kitt Peak), a mountain with particular significance to the Tohono O'odham Nation.

\section{REFERENCES}

Aihara, H., AlSayyad, Y., Ando, M., et al. 2019, PASJ, 71, 114, doi: 10.1093/pasj/psz103

Comparat, J., Richard, J., Kneib, J.-P., et al. 2015, A\&A, 575, A40, doi: 10.1051/0004-6361/201424767
DESI Collaboration, Aghamousa, A., Aguilar, J., et al. 2016, ArXiv e-prints, arXiv:1611.00036

Dey, A., Schlegel, D. J., Lang, D., et al. 2019, AJ, 157, 168, doi: 10.3847/1538-3881/ab089d

Karim, T., Lee, J. H., Eisenstein, D. J., et al. 2020,

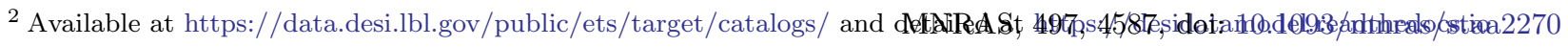


Laureijs, R., Amiaux, J., Arduini, S., et al. 2011, ArXiv e-prints, arXiv:1110.3193

Newman, J. A., Cooper, M. C., Davis, M., et al. 2013, ApJS, 208, 5, doi: 10.1088/0067-0049/208/1/5

Raichoor, A., de Mattia, A., Ross, A. J., et al. 2020, arXiv e-prints, arXiv:2007.09007
Schlegel, D. J., Finkbeiner, D. P., \& Davis, M. 1998, ApJ, 500, 525, doi: $10.1086 / 305772$

Takada, M., Ellis, R. S., Chiba, M., et al. 2014, PASJ, 66, R1, doi: 10.1093/pasj/pst019 